\title{
Focusing on the clinical diagnosis and risk of hairy polyp: A report of 7 cases
}

\author{
Zhaoyan $\mathrm{Yu}^{1}$, Zhixin $\mathrm{CaO}^{2}$, Haiyan $\mathrm{Fu}^{2}$, Haibo Wang ${ }^{1}$ \\ ${ }^{1}$ Department of Otolaryngology-Head and Neck Surgery, ${ }^{2}$ Department of Pathology, Provincial Hospital Affiliated to \\ Shandong University, Jinan, Shandong 250021, China. \\ E-mail: wang.hb7585@hotmail.com \\ Received: 26th November 2016, Revised: 7th February 2017, Accepted: 7th February 2017
}

\begin{abstract}
SUMMARY: Yu Z, Cao Z, Fu H, Wang H. Focusing on the clinical diagnosis and risk of hairy polyp: A report of 7 cases. Turk J Pediatr 2018; 60: 460-463.

The hairy polyp is a rare congenital tumor. We introspected 7 cases of hairy polyp that were operated on in our hospital. Microscopically, they all contained only ectoderm and mesoderm tissue and the surface of tumors covered skin and hair regularly. One patient died of a hemorrhage and one girl's hairy polyp occurred in nose. The result indicates that age, pedicle and thin hair are significant markers for the differential diagnosis of hairy polyp and it is important to closely follow a patient post operatively not only for airway obstruction but also hemorrhage.
\end{abstract}

Key words: hairy polyp, infant, hemorrhage, nasopharyngeal obstruction.

Hairy polyp is a rare congenital tumor, with an incidence of about $1 / 40,000 .{ }^{1}$ This kind of tumor is a mass of tissue, visible perinatally, which is composed of excessive normal tissue indigenous to a site of origin and is a tumorlike aggregation of mature polygerminal tissue anatomically. It is an inborn error of cutaneous tissue development, often in the nasopharyngeal cavity. ${ }^{2}$ Microscopically, hairy polyp is defined as a mass consisting of fatty tissue covered by skin and related structures ${ }^{3}$, which is characterized by the presence of both mesoderm and ectoderm. ${ }^{4}$ It is congenital, but found in adulthood occasionally. ${ }^{5}$ There is no clearly defined etiology. Herein, we present 7 examples of a hairy polyp to provide more data in understanding this kind of tumor.

\section{Case Reports}

We conducted a retrospective study at our hospital between January 2008 and October 2016. The study included 7 patients with hairy polyp who were all confirmed pathologically. All participants provided written informed consent.

During the initial examination, the masses were noted and the patients underwent surgery. Data inclusive of gender, age, dyspnea, dysphagia, size, location, pedicle, appearance, treatment, and pathological outcome were recorded at the time of admission. The tumor size was recorded by multiplying the length by width. The final diagnosis was based on postoperative pathological investigation. Then all patients were followed up by return visits at 1 year to 5 years after surgery. During their return visits, routine physical examinations were performed.

Patients' clinical profiles are presented in Table I. Seven patients were diagnosed with hairy polyp, with the youngest aged 18 days, the oldest 2 years, and the mean age of $308 \pm 260.45$ days. The ratio of males to females in our study was 1:6. One patient underwent nasal endoscopy, which demonstrated an epitheliumlined, pedunculated mass emanating from the right nasal septum. The other hairy polyps were all in the nasopharyngeal cavity. Five patients had tumors on the left and two on the right. The median tumor size was $5.25 \mathrm{~cm}^{2}$. (Fig.1A and Fig. 1B) There were five pedunculated hairy polyps with a mean length of $1.76 \pm 0.83 \mathrm{~cm}$ and the others' tumors grew on the mucosa of the nasopharyngeal cavity directly without pedicle. For all of them, gross morphology revealed hairy polyp as an isolated and tubular pedunculated polypoidal mass, covered by epidermis with thick, short or velvety hair. All seven patients underwent surgical resection. Microscopically, the lesions contained a clear stratified squamous 


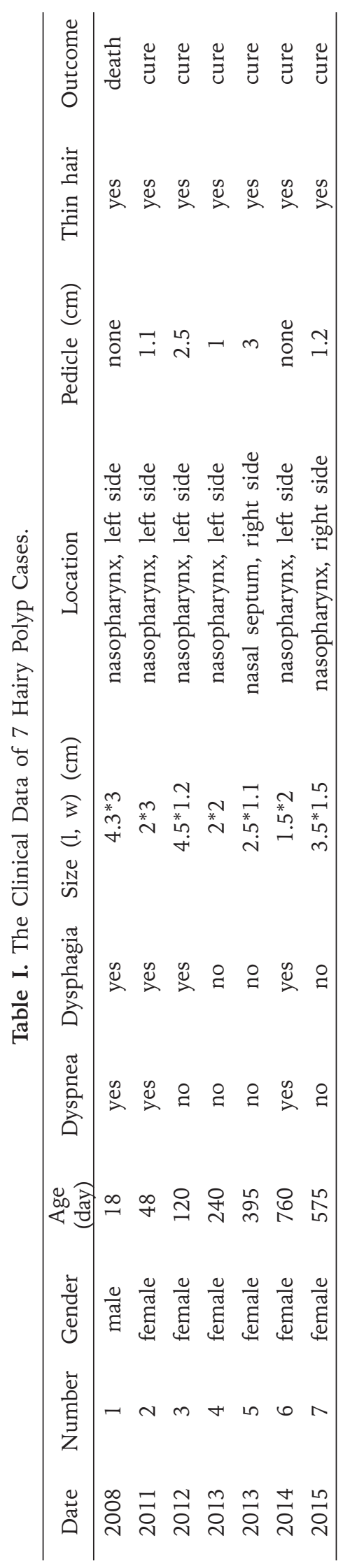

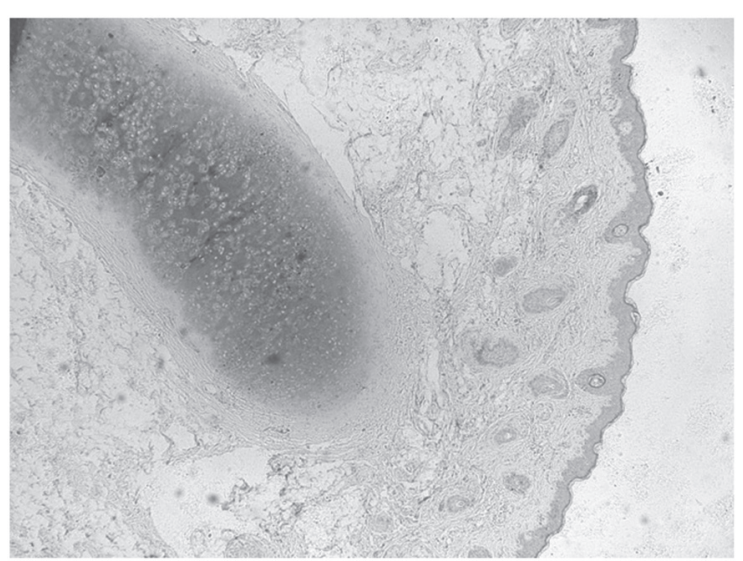

Fig. 1. (A) This photo revealed that the hairy polyp was dragged out from nasopharyngeal cavity. (B) The hairy polyp had been removed and the length was $4.5 \mathrm{~cm}$.

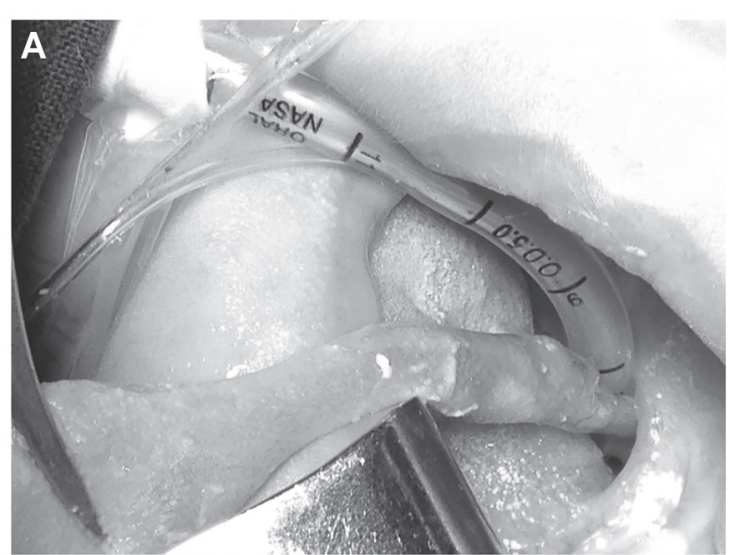

B
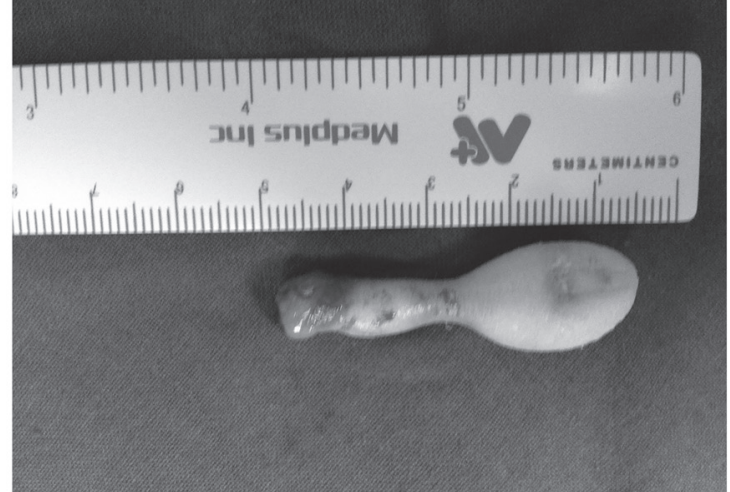

Fig. 2. This pathological picture revealed the skin and related structures covered fatty tissue and cartilage tissue. Moreover, we cannot find the endoderm tissue. (hematoxylin-eosin, 40x) 
epithelium and skin appendages, containing muscle and only one cartilage, without any tissue endoderm differentiation (Fig. 2). In clinical symptoms, there were 3 patients with a history of dyspnea and 4 patients with a history of dysphagia. Three patients suffered both dyspnea and dysphagia. None of the seven patients suffered from eustachian dysfunction or otitis media. Unfortunately, one male child died which occurred after hemorrhage and dyspnea which occurred suddenly on the fifth day after surgery. Six female patients recovered and were discharged. The longest follow-up was 5 years and the shortest 1 year. Six patients recovered completely without recurrence.

\section{Discussion}

It should be pointed out that, in aspect of differential diagnosis, hairy polyp should be firstly differentiated from hamartoma or choristoma because the gross observation of hairy polyp can be easily confused with those two disorders. The definitive diagnosis of hairy polyp, ultimately, is dependent on histopathology. Histopathologically, the mass is lined by an ectodermal component including keratinizing stratified squamous epithelium and skin appendages. The underlying core consists of adipocytes, skeletal muscle, smooth muscle and seromucinous glands, cartilage or lymphoid aggregates derived from mesoderm.

So far, complete resection is the first choice for the treatment of this disorder. Koike et al. ${ }^{6}$ considered the size of hairy polyp to be linked with morbidity and even in size smaller than $3 \mathrm{~cm}$. The larger the size of a tumor in the pharyngeal cavity, the rate of suffering a functional disorder will increase. In our case series one patient died after surgery so we do believe it is important to focus on the security of the patient during the postoperative period. Great attention must be paid to the complications following surgery in particular, hemorrhage and edema, both of which were high risk factors. It is worth reminding that the infant may not reflect nasopharyngeal bleeding in time. So when the infant repeats swallowing movements, the doctors need check whether the nasopharyngeal wound is bleeding.

All hairy polyp cases in our study were newborns or infants although certain researchers have reported hairy polyps in adults. ${ }^{7}$ Minoux's theory ${ }^{8}$ proposed that when the primitive pharyngeal cavity developed from pharyngeal arches in utero, the normal heterotopic cell-rests of one or more germ-line lineages histologically generated non-neoplastic masses, followed by delayed pluripotent cellular morphogenesis. While the cell differentiation delays rarely occur in hairy polyp, this theory may classify hairy polyps into choristoma, hamartoma and teratoma. It speculates the etiology of the disease for preventive intervention against hairy polyps.

Pedicle is not a feature contained in all hairy polyps. Tumors in 2 cases did not have pedicles. They are spherical in soft tissue or on the mucosa. The skin and mucosa are clearly demarcated to delineate the initial location of the hairy polyp, supported by regular skin. Despite similar color of the tumor and the surrounding tissue, the mucosa is devoid of hair and skin.

The female preponderance of hairy polyps is interesting and remains unexplained. In the review by Kalcioglu et al. ${ }^{9}$ they suggest the female-to-male ratio of $6: 1$ that most studies suggest has increased to 8:1. However, Dutta et al. ${ }^{2}$ found it to be 3.5:1 which is a significant deviation from earlier reports. Their data suggest 16 male patients in the last 25 years, with 10 reported in the last 7 years. Therefore, the female preponderance maybe an overestimation, probably attributed to excessive reporting in recent years. Additional molecular mechanisms involving embryonic development and literature reviews are needed to explain this interesting female preponderance.

Hairy polyp occurs on the left side typically, with an incidence of $6: 1$. This feature may be attributed to sonic hedgehog or Hox genes. ${ }^{10}$ The origin of hairy polyp can also be bilateral, which is rare, suggesting that hairy polyps may arise from two or more archaeocytes.

In conclusion, although the histopathologic examination is the gold standard, the present study indicates that age, pedicle and thin hair are significant markers for the differential diagnosis of hairy polyp and it is important to closely follow a patient post operatively not only for air way obstruction but also for hemorrhage. 


\section{REFERENCES}

1. Budenz CL, Lesperance MM, Gebarski S. Hairy polyp of the pharynx obscured on physical examination by endotracheal tube, but diagnosed on brain imaging. Pediatr Radiol 2005; 35: 1107-1109.

2. Dutta M, Roy S, Ghatak S. Naso-oropharyngeal choristoma (hairy polyps): an overview and current update on presentation, management, origin and related controversies. Eur Arch Otorhinolaryngol 2015; 272: 1047-1059.

3. Cantarella G, Gaffuri M, Pugni L, Pignataro L, Mosca F. Severe respiratory distress at birth caused by a hairy polyp of the Eustachian tube: Transoral endoscopyguided treatment. Congenit Anom (Kyoto) 2015; 55: 158-160.

4. Chaudhry AP, Lore JM, Jr., Fisher JE, Gambrino AG. So-called hairy polyps or teratoid tumors of the nasopharynx. Arch Otolaryngol 1978; 104: 517-525.
5. Kaplan Y, Ulkumen B, Gokpinar S, Senel Z. Adult nasopharyngeal hairy polyp presenting with middle ear effusion. Medical Science \& Discovery 2015; 2: 194-196.

6. Koike Y, Uchida K, Inoue $\mathrm{M}$, et al. Hairy polyp can be lethal even when small in size. Pediatr Int 2013; 55: 373-376.

7. Green VS, Pearl GS. A 24-year-old woman with a nasopharyngeal mass. Benign nasopharyngeal hairy polyp. Arch Pathol Lab Med 2006; 130: e33-e34.

8. Minoux M, Antonarakis GS, Kmita M, Duboule D, Rijli FM. Rostral and caudal pharyngeal arches share a common neural crest ground pattern. Development 2009; 136: 637-645.

9. Kalcioglu MT, Can S, Aydin NE. Unusual case of soft palate hairy polyp causing airway obstruction and review of the literature. J Pediatr Surg 2010; 45: e5-e8.

10. Tabin CJ. The key to left-right asymmetry. Cell 2006; 127: $27-32$ 